\title{
Fondi integrativi del SSN tra principi ed evoluzione
}

\author{
di Elio Borgonovi
}

Riflettere sul passato, vivere il presente e prepararsi al futuro è una regola che vale per la vita delle persone e per quella delle organizzazioni, istituzioni, aziende, corpi sociali ed economici. Se si vive il presente con la testa e lo sguardo rivolti al passato si soffre della sindrome di "Amarcord", che blocca il gusto della sfida e del cambiamento. Se si vive il presente senza prepararsi al futuro si rischiano "brutti risvegli" in un mondo che sarà cambiato e nel quale ci si troverà come stranieri. Se si vive il presente, soprattutto quello positivo, senza riflettere sul passato si rischia di pensare e progettare il futuro proponendo soluzioni e politiche fondate "sulla sabbia", "sui sogni", "sulle speranze" che, molte volte, porteranno non a un miglioramento ma a un arretramento. $\grave{E}$ il caso del SSN italiano che, nonostante tutte le differenze regionali, le debolezze, le critiche, che pur devono essere fatte, garantisce un livello quali-quantitativo di assistenza tra i migliori del mondo. Se si vive il presente pensando solo a "ciò che non va" senza la consapevolezza o la convinzione che nessuno "può cambiare il mondo" ma che "ognuno può cambiare quella piccola parte del mondo su cui ha influenza", si è prigionieri della sindrome di impotenza, immobilismo, critica fine a se stessa.

Questa regola ben si addice all'interpretazione di diversi fenomeni che caratterizzano l'attuale "stato di salute", per restare in tema, del SSN. Uno di questi aspetti riguarda il finanziamento del SSN e in particolare il rapporto tra spesa pubblica, che ha raggiunto il livello di circa 116 miliardi nel 2018, e spesa privata che ha raggiunto il livello di circa 40 miliardi nel 2018. Nonostante la spesa privata sia in gran parte dovuta a pagamenti diretti (out of pocket per circa 35 miliardi) si è acceso un dibattito sulla cosiddetta "sanità integrativa", rappresentata da fondi, casse negoziali o aziendali, società di mutuo soccorso. Secondo i dati pubblicati dal Ministero della Salute, a dicembre 2017 i fondi registrati all'apposita anagrafe erano 322, in forte aumento rispetto ai 267 del 2010 e dalle poche decine degli anni successivi al D.Lgs. 502/92 che ha definito per essi un primo set di norme organiche. Altri dati che caratterizzano il fenomeno sono: 10,6 milioni di iscritti, 8,3 milioni di prestazioni pagate direttamente o rimborsate dai fondi, 3,6 miliardi di euro, rimborso di 433 euro per assicurato, dati sicuramente aumentati dal dicembre 2017. Per un'analisi più aggiornata si rinvia all'Osservatorio sui consumi privati in sanità del CERGAS-SDA Bocconi. Secondo alcuni ricercatori e alcuni esponenti della politica, riconducibili anche a diversi partiti e movimenti, il rapido aumento dei fondi che godono di agevolazioni fiscali sarebbe una delle cause della crescente diseguaglianza in termini di livelli di assistenza garantiti. Quindi un rischio per il principio di universalità ed equità che sono fondamento del SSN. Applicando la regola di riflettere sul passato per capire il presente e prepararsi al futuro occorre partire da un brevissimo richiamo all'evoluzione del SSN negli ultimi 40 anni. Un arco temporale che può essere suddiviso in due periodi, quello della progettazione, che va dall' istituzione alla fine degli anni Novanta, e quello che può essere definito come adattamento e resilienza, che si è aperto con il nuovo secolo. Ciò consente di chiarire le relazioni causa-effetto, perché se si analizza il presente invertendo tali relazioni, le proposte per il futuro non possono che essere infondate, velleitarie, illusorie, controproducenti.

Per chi non ha vissuto o non ha memoria del passato, va ricordato che negli anni immediatamente successivi all'introduzione del SSN alcune assicurazioni lanciarono polizze specifiche per la sanità sulla base dell'ipotesi che una 
parte della popolazione avrebbe preferito questo canale rispetto al SSN. Questa ipotesi si rivelò infondata, le polizze non ebbero successo e causarono significative perdite, quello che in gergo colloquiale si chiama "bagno di sangue" su un piano economico, alle assicurazioni che quindi abbandonarono questa area di attività. Con il D.Lgs. 502/92 fu deciso di regolare il finanziamento privato tramite fondi integrativi che dovevano garantire prestazioni aggiuntive a quelle del SSN, ciò per non rompere i principi di universalità, solidarietà e, soprattutto, equità che erano e restano $i$ pilastri del SSN. Per questo tali fondi potevano usufruire di una fiscalità agevolata. Con il D.lgs. 229/99 fu ribadita la distinzione tra fondi cosiddetti DOC, che potevano usufruire della fiscalità agevolata in quanto garantivano almeno un $20 \%$ di prestazioni non previste dai LEA, e fondi che non potevano usufruire di fiscalità agevolata, in quanto prevedevano prestazioni sostitutive dei LEA.

Tuttavia negli ultimi quindici anni si è assistito a un aumento del numero di fondi per una serie di motivi tra $i$ quali si ricordano:

a) aumento dei bisogni di salute a seguito anche dell'allungamento della vita;

b) aumento dei servizi e prestazioni generati dal progresso scientifico e dall'innovazione tecnologica che non erano recepiti dai LEA;

c) mancato adeguamento dei LEA dal 2001, con l'aggravante che quelli approvati nel 2017 non sono stati emanati in mancanza di definizione dei relativi $D R G$;

d) presenza di liste d'attesa in molte realtà che hanno spinto una parte della popolazione a trovare soluzioni alternative tra le quali i fondi;

e) inserimento dei fondi salute nell'ambito delle nuove tendenze di cosiddetta ristrutturazione del welfare ed evoluzione verso il welfare negoziale e aziendale, con l'incentivazione fiscale prevista da varie leggi di stabilità/ di bilancio.

Questa evoluzione ha portato a una situazione che può essere cosi sintetizzata:

- quota del finanziamento pubblico pari al $74 \%$ della spesa totale, spesa privata out of pocket pari al 21-22\%, spesa privata intermediata (fondi, casse, assicurazioni) pari al 4-5\%: questa situazione è anomala rispetto ad altri Paesi nei quali la quota di spesa privata intermediata è elevata rispetto a quella out of pocket;

- crescita numerica dei fondi registrati dall'Osservatorio previsto presso il Ministero della Salute, aumento del numero degli iscritti ai fondi e casse, ma complessivamente spesa privata intermediata limitata rispetto alla spesa out of pocket;

- sviluppo dei fondi non governato e con offerte non sempre trasparenti.

Si pone quindi l'esigenza di pensare a introdurre criteri e principi per governare la spesa privata, sostenuta direttamente dalle famiglie o intermediata dai fondi, cercando di evitare o ridurre il rischio che lo sviluppo "non governato" degli ultimi anni porti a un abbandono non dichiarato dei principi fondanti del SSN, copertura universale, solidarietà ed equità. Inoltre una nuova governance in grado di spostare parte della spesa privata out of pocket al circuito della sanità intermediata consentirebbe anche di combattere l'evasione che si stima essere elevata nel circuito del pagamento diretto delle famiglie ai professionisti.

Una corretta risposta all'esigenza di un nuovo sistema di regole per i fondi integrativi deve basarsi sulla risposta alle seguenti domande.

a) È prevedibile che in futuro vi possa essere un significativo aumento della quota di finanziamento pubblico per la tutela della salute come percentuale del PIL? La risposta è sicuramente no.

b) L'aumento della domanda di salute potrà trovare risposta in un adeguamento dei LEA in tempi ragionevoli? Anche in questo caso è facilmente prevedibile una risposta negativa. 
c) Anche un significativo recupero di appropriatezza, efficacia, efficienza del SSN, comunque auspicabili e da perseguire con decisione, potranno consentire di dare risposte soddisfacenti alle evoluzioni dei bisogni? Ancora una volta la risposta sembra essere negativa.

Di conseguenza, un nuovo quadro di regole organiche per la sanità intermediata è auspicabile come soluzione realistica per perseguire i principi di universalità, solidarietà tramite fiscalità generale ed equità, un secondo pilastro basato su solidarietà volontaria e mutualità, un terzo pilastro costituito da assicurazioni private che applicano solo il principio di mutualità e non solidarietà. In assenza di un nuovo quadro organico di regole il sistema della sanità intermediata evolverebbe in modo frammentario accentuando le disuguaglianze non desiderabili, invece che governare "differenze" riconducibili a consapevoli scelte delle persone, delle aziende, di gruppi sociali (gli aderenti ai fondi). Peraltro parlare delle problematiche dei fondi integrativi separatamente da una revisione organica dell'impianto del SSN aumenterebbe la situazione di frammentarietà e disorganicità del sistema.

La riflessione su fondi negoziali, casse aziendali, SMS (sanità intermediata) al fine di elaborare proposte di intervento deve basarsi su dati e una chiara comprensione delle relazioni domanda-offerta, onde evitare interventi che invece di portare a miglioramenti possono peggiorare la situazione. Infatti, auspici, buone intenzioni, dichiarazioni di intenti non sono sufficienti e anzi possono essere controproducenti se non sono fondati sulla conoscenza dei reali comportamenti delle persone e dei sistemi sociali. Innanzitutto va ricordato che la sanità intermediata si è sviluppata a causa dell' indebolita capacità del SSN di rispondere ai bisogni. Sarebbe drammatico invertire la comprensione delle relazioni causa-effetto. Infatti lo sviluppo della sanità intermediata di tipo solidaristico è la conseguenza e non la causa della progressiva incapacità del SSN di garantire un universalismo esteso. Ciò è dimostrato dal fatto che gli anni Novanta sono stati caratterizzati da un numero limitato di fondi, poche decine, che è aumentato progressivamente con il nuovo secolo e soprattutto negli ultimi dieci anni.

In secondo luogo è errato ritenere che i fondi, le casse aziendali, le SMS e perfino le assicurazioni private siano la causa prima di "consumismo sanitario". Studi e ricerche che risalgono agli anni Cinquanta e Sessanta hanno evidenziato che il "consumismo sanitario" è alimentato prevalentemente dall'offerta, ossia dal numero di medici e di strutture. Diversamente da un sistema fondato prevalentemente e in modo esteso sulle assicurazioni private, la sanità intermediata che costituisce il secondo pilastro del welfare ha semmai l'esigenza opposta, ossia quella di contenere il consumismo sanitario e controllare i comportamenti delle strutture di offerta. In questo caso il "terzo pagante" privato e solidaristico è in genere più forte del "terzo pagante" costituito dal SSN, in quanto eccessive richieste di prestazioni comporterebbero l'aumento dei contributi (delle aziende o degli individui) o renderebbero più difficile mantenere l'equilibrio di bilancio. In Italia si poteva parlare di spinta al consumismo sanitario nel periodo in cui vi era un numero elevato di medici rispetto alla popolazione (anni Settanta, Ottanta e in parte Novanta). Oggi la carenza di medici, infermieri, altro personale di assistenza da un lato e l'aumento strutturale dei bisogni dall'altro (allungamento della vita, patologie degenerative, cronicità di vario tipo ecc.) pongono un diverso problema, quello di razionalizzare l'offerta con azioni finalizzate a responsabilizzare maggiormente sulla appropriatezza, efficacia, efficienza. Se di spinte al consumismo si vuole parlare, esse riguardano soprattutto il circuito dei pagamenti diretti da parte dei pazienti che hanno scarsa capacità negoziale nei confronti degli erogatori, anche se oggi possono far riferimento a situazioni di "competizione" soprattutto per le prestazioni cosiddette "low cost".

In terzo luogo va sottolineato che la situazione italiana non è anomala in termini di percentuale della popolazione che fa riferimento alla sanità intermediata. Da un'analisi comparativa condotta dall'Osservatorio sui consumi privati in sanità (OCPS) del CERGS-SDA Bocconi risulta che nel 2016 il 18,7\% della popolazione faceva riferimento alla sanità intermediata, percentuale in linea con Gran Bretagna (16\%) Spagna (22,5\%), Portogallo (17\%), per non parlare della Francia che ha forme di copertura privata obbligatoria per alcune esigenze cui aderisce il 93\% della popolazione.

In quarto luogo sarebbe illusorio e controproducente ritenere che eliminare la fiscalità agevolata per le forme di sanità integrativa/intermediata di tipo negoziale potrebbe rendere disponibili maggiori finanziamenti per il SSN. Infatti 
le eventuali maggiori entrate sarebbero riassorbite nell'ambito dei vincoli di finanza pubblica e con ogni probabilità non sarebbero destinate a un aumento del fondo sanitario nazionale. Peraltro si parla di togliere agevolazioni riferite a 3,6 o al massimo 4 miliardi di finanziamenti, ben poca cosa rispetto al potenziale recupero derivante da un'efficace lotta all'evasione ottenibile con il passaggio da spesa out of pocket a spesa intermediata. Una normativa che eliminasse la possibilità di sostituire maggiori retribuzioni per aumento di produttività o comunque di tipo premiale con fondi salute determinerebbe un aumento del "cuneo fiscale" che invece andrebbe ridotto per rilanciare la crescita. Infatti la tassazione delle maggiori retribuzioni corrisposte ai lavoratori aumenterebbe il divario tra costo per le imprese e retribuzione netta con un limitato aumento delle entrate tributarie, dell'ordine di poche centinaia di milioni.

In quinto luogo politiche che rischiano di essere penalizzanti per forme di sanità integrativa/intermediata determinerebbero un allargamento del malessere di 12 milioni di italiani, in forte aumento ogni anno, che oggi utilizzano questo canale per accesso a forme di "prestazioni leggere", oggi erogate da privati a costi molto bassi, o di "prestazioni urgenti" (accertamenti diagnostici e di controllo) non garantite dalle lunghe liste d'attesa. Creare difficoltà alla operatività dei fondi, casse aziendali, SMS avrebbe l'effetto di aumentare la pressione sulle strutture del SSN con un ulteriore aumento delle liste di attesa che possono essere superate solo da chi ha elevate disponibilità di reddito. Si creerebbero difficoltà per gli individui e le famiglie che costituiscono la cosiddetta classe media della popolazione, senza vantaggi per i gruppi poveri e fragili. Anzi, potrebbe aiutare a mitigare l'effetto di "marginalizzazione" e di "bassa accessibilità ai servizi" dei gruppi poveri e fragili il consentire e favorire che una parte limitata dei sostegni economici alla povertà (REI, reddito di cittadinanza, altre che in futuro fossero adottate) possa essere destinata a forme di sanità integrativa/intermediata.

In definitiva il bilanciamento tra vantaggi e svantaggi delle forme di sanità integrativa/intermediata può essere ottenuto se i due circuiti, quello del SSN e dei LEA e quello del secondo pilastro del welfare, invece di essere considerati contrapposti saranno considerati due motori che concorrono a un più elevato livello quantitativo e qualitativo di risposte ai bisogni reali di salute. Il rilancio di una nuova fase progettuale per il SSN dovrà prevedere regole che tengano conto anche dei vantaggi e dei rischi strutturali delle forme di sanità integrativa/intermediata. Tra $i$ vantaggi si ricordano:

- l'aumento delle risorse complessive destinate alla sanità (alleggerimento della crisi fiscale e contributiva);

- un più ampio e rapido accesso alle cure;

- una maggiore opportunità di scelta e stimolo alla competitività del pubblico;

- una migliore qualità ed efficienza del sistema nel suo complesso;

- il riaggiustamento delle responsabilità tra individui e collettività.

Le perplessità o i rischi riguardano invece:

- l'erosione delle basi di consenso dei sistemi pubblici (circoli viziosi);

- l'inevitabile creazione di un sistema duale (minacce all'equità);

- alcuni rischi di "relazioni improprie" quasi inevitabile quando pubblico e privato condividono i sistemi di offerta (aumento dei costi nel pubblico, impatto sulle priorità e processi di razionamento, comportamento dei professionisti interessati a dirottare parte della domanda dal circuito dei servizi istituzionali a quello delle prestazioni private coperte dai fondi).

Peraltro, una normativa organica della sanità intermediata, nell'ambito di una più generale riforma del SSN, appare non più procrastinabile per rispondere agli obiettivi comunitari di cui al "Solidarity in Health. Reducing Health Inequalities in EU" (Paper della Commission Communication) del 20 ottobre 2009 e con i principi di libera circolazione con riguardo all' accesso alle cure dei cittadini europei. Una normativa organica, che mantenga la 
copertura universale, garantisca la sostenibilità sociale ed economica, consenta la libera scelta di cittadini e famiglie sull' allocazione del proprio reddito deve perciò ispirarsi ai principi di seguito elencati.

Sviluppo di una sanità privata intermediata, che sia in parte complementare e in parte sostitutiva a quella pubblica aperta a tutti, imperniata sulle aggregazioni di persone, che consenta di razionalizzare la spesa, sfruttare i vantaggi del raggruppamento in termini di forza contrattuale nei confronti delle strutture di offerta, di mutualità e di solidarietà tra gli aderenti. La componente sostitutiva/sovrapponibile ai LEA potrà essere definita come percentuale della spesa dei fondi modulabile nei vari periodi storici e dovrà riguardare quei bisogni per i quali il SSN presenta maggiori difficoltà di risposta.

Definizione di regole e di indirizzi che consentano di governare diverse forme di sanità privata intermediata con la finalità di individuare e definire aree di bisogni e tipologie di servizi più efficaci per promuovere l'appropriatezza e ridurre le inefficienze del SSN. Una normativa organica può prevedere regole idonee a evitare che la solidarietà volontaria garantita da fondi, casse aziendali, SMS abbia effetti negativi sui livelli assistenziali garantiti dal SSN. Il terzo pilastro, circuito privato-privato finanziato da spesa out of pocket o sottoscrizione di assicurazioni, consentirebbe alle famiglie e agli individui la libertà di utilizzare il proprio reddito secondo le proprie preferenze rispetto ad altri bisogni privati. Un sistema di regole, per essere efficace e credibile, dovrebbe prevedere le seguenti funzioni:

a) rafforzamento della funzione di programmazione, monitoraggio e vigilanza sui diversi soggetti pubblici, privati e misti che operano nel sistema di tutela della salute;

b) identificazione dei livelli di solidarietà e delle garanzie di solvibilità che devono essere soddisfatti per godere di vantaggi normativi e fiscali;

c) riconoscimento degli incentivi fiscali sia ai gruppi sia agli individui che si aggregano con forme giuridiche che rispettano $i$ criteri di solidarietà e di solvibilità individuati, superando l'attuale discriminazione fra fondi doc e fondi non doc, che non risponde più alla logica del 1992 e del 1999 che l'aveva ispirata;

d) flessibilità del sistema poiché l'approvazione dei nuovi LEA è destinata a creare problemi, in quanto l'ampliamento delle prestazioni teoricamente coperte, collegata al permanere di vincoli del fondo sanitario nazionale (che si prevede non aumenterà in modo significativo nei prossimi anni) creerà crescenti tensioni tra LEA teorici e LEA concretamente garantiti.

Oggi appare necessaria una riforma organica per regolare in modo sinergico, integrato e innovativo le diverse relazioni che si sono sviluppate nel sistema, in particolare:

- finanziamenti pubblici a erogatori pubblici;

- finanziamenti pubblici a erogatori privati accreditati/convenzionati;

- finanziamenti privati intermediati (fondi, casse, SMS, assicurazioni) a erogatori privati;

- finanziamenti privati out of pocket a erogatori privati;

- finanziamenti privati (intermediati o out of pocket) a erogatori pubblici o privati accreditati (es. convenzioni con ospedali o IRCCS pubblici, finanziamento di prestazioni intra moenia in strutture pubbliche).

Un quadro normativo organico consentirebbe di utilizzare la diversificazione e l'integrazione dei canali di finanziamento come strumento per affrontare con soluzioni flessibili le esigenze derivanti dalla continua innovazione nel campo della tutela della salute e dei comportamenti dei pazienti.

Entrando in maggiore dettaglio, il sistema di governance del SSN riferito alla problematica della "sanità intermediata" dovrebbe bassarsi sui seguenti elementi:

- il rafforzamento dei poteri di indirizzo e di programmazione del Ministero della Salute;

- la definizione di osservatori per la raccolta di dati e di informazioni affidabili per identificare le aree di bisogni e 
le tipologie di prestazioni per le quali appare meno efficace e tempestiva la capacità di risposta del SSN: funzione che spetta al Ministero della Salute e ai propri organi tecnici (ISS, Agenas ecc.) in accordo con la Conferenza Stato-Regioni;

- la definizione di schemi omogenei e comparabili per la raccolta dei dati, cui dovranno attenersi sia gli erogatori pubblici sia quelli privati, in modo da consentire analisi comparative di tipo longitudinale (nel tempo) e spaziale (comparazione tra Regioni);

- la consultazione delle Regioni e dei vari soggetti pubblici e privati che operano nel sistema;

- il mantenimento della tipologia e della percentuale di prestazioni che i fondi dovranno rispettare per usufruire del trattamento agevolato per tempi medio-lunghi, almeno 3-5 anni, onde consentire agli stessi di definire le proprie politiche nei confronti degli associati. Ciò può essere ottenuto rivedendo la percentuale che i fondi con fiscalità agevolata devono obbligatoriamente destinare a certe coperture;

- l'introduzione di un sistema di vigilanza sui soggetti della sanità intermediata con riguardo al rispetto dei criteri e dei parametri di monitoraggio della solvibilità a garanzia degli associati.

L'insieme dei soggetti che rappresentano la sanità privata intermediata (fondi, casse, SMS, compagnie di assicurazione) dovrebbe essere sottoposto alla vigilanza attribuita a una specifica autorità con le seguenti funzioni:

- verifica della corrispondenza degli statuti alla normativa riguardante SMS, fondi, casse aziendali;

- monitoraggio del rispetto e del mantenimento nel tempo dei requisiti richiesti per godere delle agevolazioni normative e fiscali;

- valutazione della sostenibilità economica e finanziaria posta a garanzia degli associati e della finanza pubblica;

- definizione di regolamenti eventualmente richiesti dalla applicazione della normativa quadro sulla sanità intermediata, con riferimento ai diversi soggetti, fondi negoziali, casse aziendali, SMS;

- definizione di regolamenti per l'esercizio delle funzioni ispettive e sanzionatorie;

- rispetto degli obblighi di informazione, trasparenza e accountability nei confronti delle parti sociali, degli associati e di altri soggetti interessati alla loro attività.

In conclusione, è possibile sostenere che lo sviluppo della sanità privata intermediata, ponendosi come secondo pilastro nel sistema generale della tutela della salute, può rappresentare, laddove a essa si riconosca una funzione non solo complementare ma anche sostitutiva/sovrapponibile ai LEA, un rilevante strumento per la sostenibilità della sanità pubblica. Infatti consentirebbe di far affluire al sistema risorse private in forma solidaristica che, tramite l'Osservatorio sulle dinamiche dei consumi in precedenza richiamato, può prevenire anche forme di consumismo sanitario. Riprendendo la regola aurea della vita con cui si è iniziato questo editoriale si può dire che dopo la fase di progettazione del SSN (anni Ottanta e Novanta), quella della flessibilità, adattamento, resilienza (ultimi due decenni del nuovo secolo), occorre una nuova progettualità che sia in grado di coniugare i principi di universalità, solidarietà, equità nel contesto attuale e in quello che si va delineando per il futuro. Esistono due modi per distruggere un sistema sociale: rinnegare i principi fondanti e proporne dei nuovi, oppure applicare in modo rigido principi validi in astratto. Non si intende teorizzare un "sano pragmatismo" ma evitare che anche con riferimento al SSN prevalga quanto ricordato dal Cardinal Gianfranco Ravasi nel suo recente volume Breviario dei nostri giorni: "I principi? Quelli non servono. Sono cose acquisite, vestiti, stracci ornamentali che volano via alla prima scrollata vigorosa". Dove sono necessarie risorse la scrollata può venire dai vincoli di finanza pubblica. 